\title{
A LINGUISTIC APPROACH TO THE ANALYSIS OF ACCELEROMETER DATA FOR GAIT ANALYSIS
}

\author{
Anita Sant'Anna \\ School of Information Science, \\ Computer and Electrical Engineering \\ Halmstad Suniversity - Sweden \\ email: anita.santanna@hh.se
}

\author{
Nicholas Wickström \\ School of Information Science, \\ Computer and Electrical Engineering \\ Halmstad Suniversity - Sweden \\ email: nicholas.wickstrom@hh.se
}

\begin{abstract}
There is evidence that many cognitive conditions affect the human motor system. Gait analysis has lately been used as a means of studying this physical-cognitive correlation. The development of gait analysis systems, able to record and analyze gait during normal daily activities and in uncontrolled environment, is an important addition to this area of research. Lately, linguistic approaches have been studied as means to achieve activity classification from vision sensors. The present work aims to extend the linguistic approach to achieve quantitative analysis of gait from accelerometer data. The proposed method can be used to extend the Human Activity Language framework to include the analysis of inertial sensors such as accelerometers. Results show that the proposed method is more accurate and robust than previous methods and can be used to extract a number of clinically relevant gait measurements. A novel symmetry index is presented to exemplify how the proposed method is able to extract more information from accelerometer signals than previous methods.
\end{abstract}

\section{KEY WORDS}

Gait analysis, accelerometers, non-invasive measurements

\section{Introduction}

Understanding the relationship between physical performance and cognitive condition is important for the development of motion-based cognitive assessment systems. Such systems could improve the diagnosis and assessment of cognitive conditions given that movements are easier to record and analyze than the workings of the brain. Sometimes, this physical-cognitive correlation is clear, e.g. Parkinson's Disease. However, in general, this link is subtle and hard to observe. Gait analysis studies have uncovered correlations between gait measurements and the onset of dementia and other cognitive impairments [1]. Further developments in this research area are needed, and they depend on tools for recording and analyzing human motion data.

Recent works in human motion analysis have explored the use of linguistic approaches, based on symbolization and subsequent analysis of symbol patterns and distributions. Drawing a parallel with natural languages, the symbols correspond to letters and the different movements are expressed by different words and sentences, which can be created by combining symbols according to certain grammar rules. Guerra-Filho et al. [2] have developed a framework for the Human Activity Language (HAL), which expresses how the required grammar can be extracted from symbolized data. The HAL implementation suggested by Guerra-Filho et al. was based on motion capture data.

Although motion capture systems can provide accurate description of movements, they are fixed to a certain environment and can only be operated by specially trained personnel. These systems cannot accurately capture normal daily activities in uncontrolled environments. Therefore, unobtrusive, wearable systems are important for the analysis of movements in "real-life". These wearable systems are normally composed of inertial sensors such as accelerometers and gyroscopes.

The present work applies a linguistic approach to analyze accelerometer data for gait analysis. The contributions of this work include a segmentation technique that can be used to adapt HAL to inertial sensor data, extending its usage to wearable systems. The proposed method achieves quantitative analysis of gait from symbolized data, as opposed to previous linguistic approaches focused on activity classification. The proposed method is used to accurately extract heel-strike and toe-off events from the accelerometer data. In addition, this paper exemplifies with a new gait symmetry index how the linguistic approach can extract more information from the acceleration signal than previous works.

\section{Related Work}

Gait analysis has brought forth some evidence of the link between physical and cognitive condition. For example, slower walking speed due to decreased stride length and increased double support time, and step-to-step variability have been shown to differ between patients with dementia and healthy subjects [1, 3]. Also, Verghese et al. [4] conducted a five-year longitudinal study and determined that quantitative gait measurements related to pace, rhythm, and variability can predict future risk of cognitive decline and dementia in initially non-demented older adults. 
The state of the art gait analysis (kinematics) methods make use of motion capture systems, ground force reaction plates, and elaborate software to reconstruct a complete 3D trajectory of the subject's body [5]. From this model any quantitative gait measure can be extracted. However, these systems are fixed to a certain environment (room) and can, typically, only analyze a few consecutive steps at a time. In addition, patients may act differently under such controlled situations and certain "real life" episodes may not be observed.

Therefore, the development of wearable sensors, able to continuously record and assess activities in uncontrolled environments, is an important addition to this research field. Previous works on wearable activity monitoring systems have mostly employed inertial sensors such as accelerometers $[6,7]$, and gyroscopes $[8,9]$. The present work considers accelerometers as motion sensors due to their low cost, low power consumption and small size.

Previous gait analysis studies using accelerometers have mainly focused on either the identification of gait phases, or the classification of walking patterns. Identification of gait phases is normally achieved through detection of particular events in time such as heel-strike (HS) and toe-off (TO), e.g. [10]. These studies are typically based on techniques such as thresholding or peak detection, e.g. $[11,6]$. Supervised machine learning techniques, such as Artificial Neural Networks (ANN), have also been used, e.g. [12]. The methods concerned with identifying gait phases normally convey only temporal information about the signal and do not provide information about how the subject's feet are moving through space.

The other group of methods, on the other hand, aims to classify walking patterns based on certain dynamics of the walk: walking cadence, if the subject is walking up or down stairs, running, e.g. [13]. These studies normally involve methods based on sliding-window statistics [14], time-frequency analysis [15], wavelet decomposition [16], and/or ANN [17]. This class of methods is able to describe, to a certain extent, how the subject is walking. However, the phases of gait are overlooked and the information obtained from such methods is insufficient for clinical gait analysis applications. In contrast to both groups of previous studies, the present work aims to simultaneously extract both temporal events and dynamic characteristics in order to perform gait analysis for clinical applications.

Many studies have investigated the use of linguistic approaches to human motion analysis. Linguistic approaches are based on symbolization followed by an analysis of how different symbols are combined according different activities. An important practical advantage of working with symbols is that the efficiency of numerical computations is greatly improved, i.e. compression. Also, symbolic data is often less sensitive to measurement noise. Although it is not obvious that a symbolic approach is useful when dealing with systems having continuous state spaces, many previous works have used symbolic transformation of continuous data with great success [18]. Another advantage of

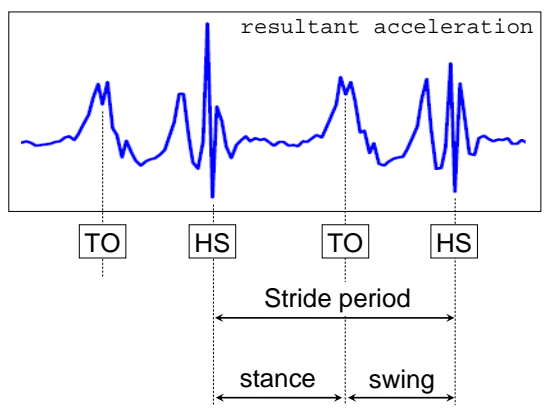

Figure 1. Example of accelerometer gait data and the corresponding phases of gait.

linguistic approaches is the possibility to use context-based analysis. A classic example of context-based analysis is Optical Character Recognition, where the uncertain individually classified characters in a word are checked against a list of possible words. A similar context-based analysis can be performed on motion data if the possible symbol patterns are identified.

Previous linguistic approaches have expressed visual data in terms of symbols, then classified patterns into one of a few pre-defined labeled patterns (activities). e.g. [19, 20, 21]. Guerra-Filho el al. [2] went one step further and created a framework for the Human Activity Language (HAL), which concerns the representation of human movement in terms of atomic visuo-motor primitives. They introduced the concept of kinetology, the phonology of human movement, and illustrated how HAL can describe a number of different activities using motion capture data. The present work contributes to this research area in two main ways: it applies a linguistic approach to analyze accelerometer data; and it aims at the quantitative analysis of gait patterns, instead of activity classification.

\section{Method}

The method proposed here aims to extract not only temporal information about HS and TO instances, but also dynamic information about walking patterns from acceleration signals. This is achieved through symbolization of the signal, and analysis of the context and distribution of each symbol. An example of typical accelerometer gait data and the phases of gait in illustrated in Figure 1.

\subsection{Symbolization}

The symbolization of the signal is illustrated in Figure 2. After filtering the acceleration signal with a low-pass FIR filter with cut-off frequency $20 \mathrm{~Hz}$, the resultant acceleration is computed: $A_{\text {res }}=\sqrt{A_{x}^{2}+A_{z}^{2}}$, where $A_{x}$ and $A_{z}$ are accelerations in the accelerometer's local coordinate system. The "sideways" acceleration axis was not considered because the subjects walked a straight line (See Section 4 for details about the experimental setup). The acceleration signal was segmented with a piecewise linear ac- 


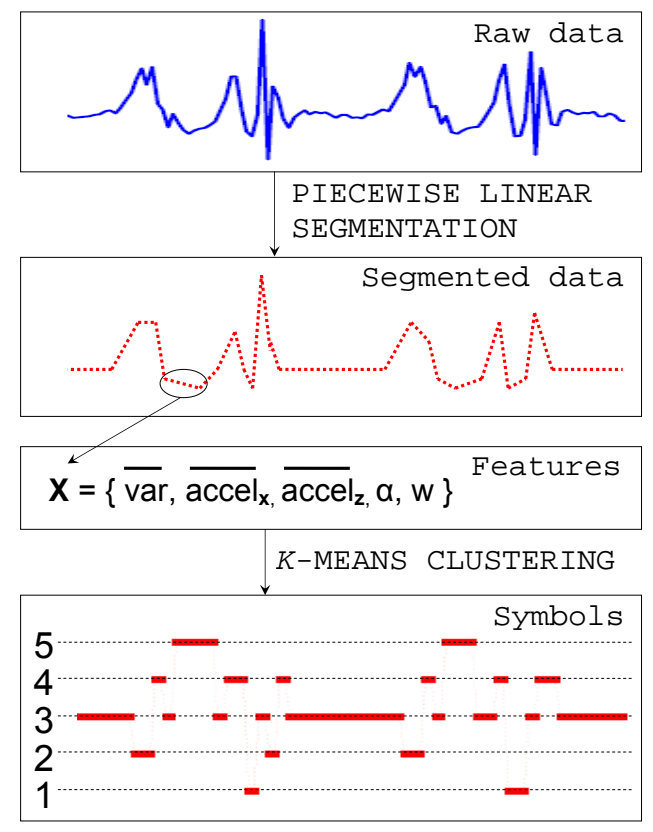

Figure 2. Symbolization. Graphical representation of the steps taken towards symbolization of the signal.

celeration method, implemented as described in [22]. The features extracted from each segment were: mean segment variance of the resultant acceleration, $v \bar{a} r$; mean segment acceleration on both axes, accel $l_{x}$ and accel $_{z}$; the tangent of the angle between the approximated line segment and the horizontal axis, $\alpha$; and the number of samples in the segment, $w$.

The segment features were standardized (zero mean and unit standard deviation) and divided into different groups using k-means clustering with randomized initial conditions. Clustering was performed considering from 2 to 10 clusters. The optimum number of clusters was chosen based on the minimum Davies-Bouldin index [23]. The limit of 10 clusters was chosen because most of the initial trials resulted in less than 10 clusters. A unique symbol was assigned to the segments within each cluster. For simplicity, the symbols are integers contained in the interval between 1 and the number of clusters.

\subsection{Context Analysis}

The physical characteristics of the system are reflected on the symbolic data as certain symbol sequences or symbol distributions. These observations were incorporated into the model in order to find the symbols corresponding to HS and TO. The algorithm is divided into three main steps: finding relevant symbols, hypotheses testing, and estimating "likeliness", as illustrated in Figure 3.

Relevant symbols are symbols which are likely to express striking characteristics of the original signal, such as HS and TO, and are expected to appear approximately once every cycle. Symbols with period similar to or half of

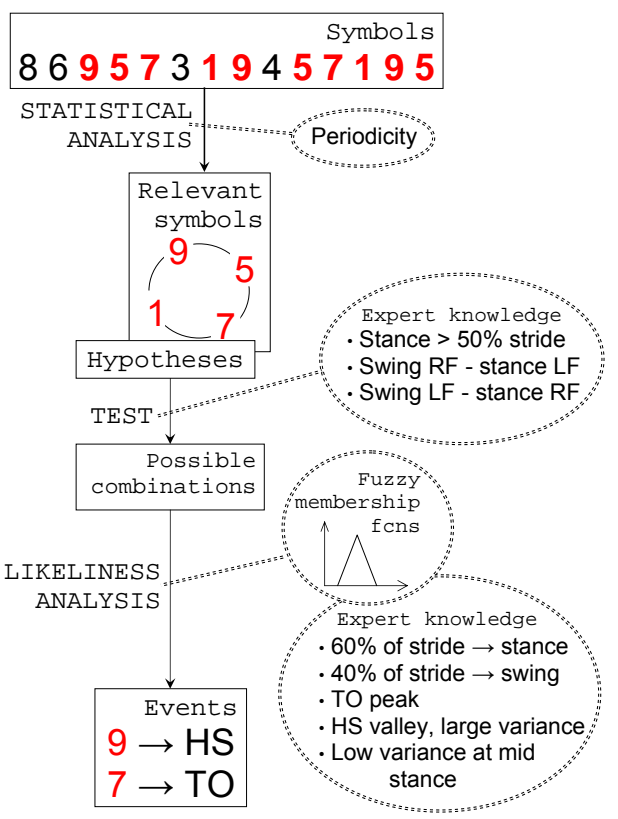

Figure 3. Context analysis. Graphical representation of the steps taken towards context analysis of the signal.

the estimated stride period are considered relevant symbols. For every possible pairwise combination of relevant symbols, a hypothesis is created that one symbol corresponds to HS and the other to TO. These hypotheses are tested according to the assumption that stance lasts over $50 \%$ of the stride time, and swing of one foot can only take place during stance of the other foot. The hypotheses which fail to comply to these assumptions are discarded. The remaining hypotheses are ranked according to how likely their are to be true based on the following gait characteristics:

1. During normal walk, approximately $60 \%$ of the total stride time corresponds to stance;

2. TO is reflected on the resultant acceleration signal as a peak;

3. HS is reflected on the resultant acceleration signal as a valley and large variance;

4. The foot moves the least at mid-stance;

Each characteristic is represented by a fuzzy membership function which maps measures such as average symbol acceleration and variance to a likeliness value. The most likely hypothesis is considered true, and HS and TO are identified. The reader is referred to [24] for more details.

\subsection{Gait Measurements}

Once HS and TO have been identified, a number of clinically relevant gait measurements can be computed [8]. This paper will focus on measures of gait symmetry. A commonly used clinical measure of symmetry is the relative difference between stride times of both feet: $S I=$ $\frac{T_{R}-T_{L}}{\frac{1}{2}\left(T_{R}+T_{L}\right)} 100$, where $T_{R}$ is the average stride time for the 


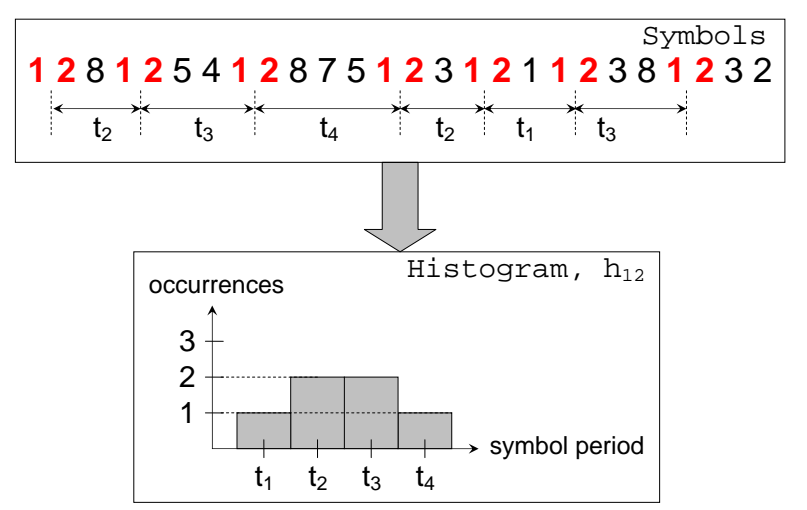

Figure 4. Transition histogram. Example of how to compute the histogram of transition periods for transition 1-2. The time elapsed between two consecutive transitions is calculated and the corresponding histogram bin is incremented.

right foot and $T_{L}$ is the average stride time for the left foot [25].

According to $S I$, the closer the absolute value is to zero, the more symmetric the walk. Although a negative value indicates that the left foot is slower than the right foot, a slower stride time does not indicate a more abnormal movement and the "affected" side cannot be determined. The value for this index ranges from -200 to 200 and, in practice, a correspondence between this index and quality of gait is unclear. This measure of symmetry only takes into account the average stride time for each foot. If the subject limps but manages to keep the same cadence, the $S I$ index will not consider this to be an asymmetric walk.

The symmetry index described above takes only temporal information into account. A more informative index can be computed from the symbolized data. After the segments are clustered into $Z$ symbols, $\boldsymbol{S}=\left\{S_{1}, S_{2}, \ldots S_{Z}\right\}$, the symbol sequence corresponding to the original data is analyzed in terms of its transition periods. Transition $i j$ refers to the moment when symbol $i$ ends and symbol $j$ begins. Histograms of the symbol transition periods are calculated for all possible transitions, i.e. $\left\{\left(S_{1}, S_{2}\right),\left(S_{1}, S_{2}\right), \ldots\left(S_{i}, S_{j}\right), \ldots\left(S_{Z}, S_{Z}\right)\right\}$. The calculus of transition histograms is exemplified in Figure 4. The symmetry index based on the transition histograms, $S I_{\text {symb }}$, is computed according to:

$$
S I_{\text {symb }}=\frac{\sum_{i, j=1}^{Z} \frac{1}{n_{i j}} \sum_{k=1}^{K}\left|h_{R i j}(k)-h_{L i j}(k)\right|}{\sum_{i, j=1}^{Z} \frac{1}{n_{i j}} \sum_{k=1}^{K}\left|h_{R i j}(k)+h_{L i j}(k)\right|} 100,
$$

where $Z$ is the number of symbols; $K$ is the number of bins in the histograms; $n_{i j}$ is the number of non-empty histogram bins for transition $i j ; h_{R i j}(k)$ is the normalized value for bin $k$ in the transition histogram $i j$ for the right foot; and $h_{L i j}(k)$ is the normalized value for bin $k$ in the transition histogram $i j$ for the left foot.

The $S I_{\text {symb }}$ ranges from 0 to 100 , where 0 means to-
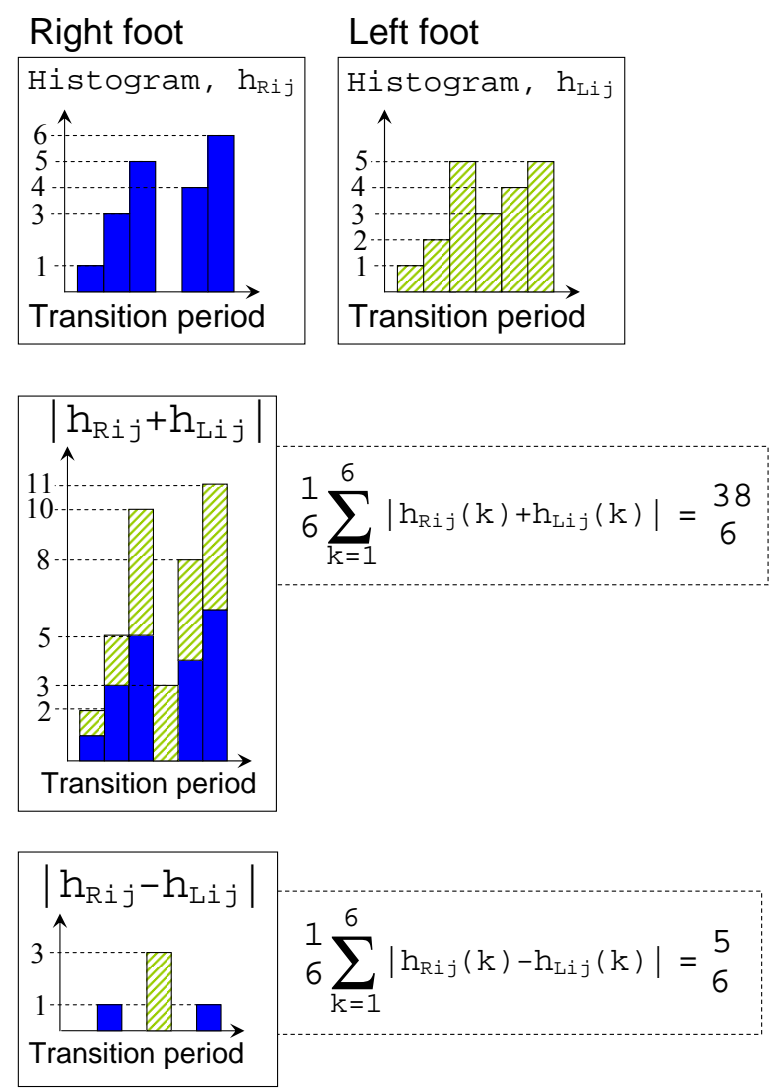

Figure 5. Histogram analysis. Example of histogram analysis for one particular pair of transition histograms. The symmetry index is a combination of this analysis for all transitions.

tal symmetry and 100 means complete asymmetry. $S I_{\text {symb }}$ takes into account not only temporal information but also the dynamics of the movement. $S I_{\text {symb }}$ can be interpreted as a comparison between the distributions of the transition periods of both feet. Figure 5 illustrates the histogram analysis for one pair of transition histograms. The $S I_{\text {symb }}$ index demonstrates one way in which the symbol abstraction used in this method can be used to extract meaningful dynamics information from the sensor data.

\section{Experimental Set-up}

Gait acceleration data was collected in order to investigate the use of the proposed method for gait analysis. The hardware used for the experiments consisted of:

- Two SHIMMER sensor nodes [26] each equipped with one tri-axial accelerometer, which is sampled at $50 \mathrm{~Hz}$ and the data is streamed continuously via Bluetooth to a nearby computer.

- A six-meter-long Gold Gait Rite pressure sensitive mat [27], sampling at $60 \mathrm{~Hz}$. The Gait Rite has its own software for detecting HS, TO and other temporal gait measurements from the pressure sensitive mat. The Gait Rite is only used to provide a reference signal, it is not part of the proposed method. 
Six subjects participated in the experiments. The subjects had the SHIMMER nodes attached to both shins, close to the ankles. When the subject was standing still, the $z$ axis of the accelerometer corresponds to the vertical axis, and the $x$ axis corresponds to the horizontal axis tangential to the subiect's sagittal plane. as illustrated in Figure 6.

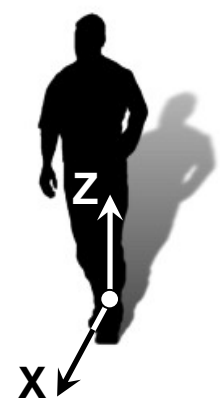

Figure 6. Accelerometer's local coordinate system

The subjects were asked to walk a straight line on the pressure mat according to three different instructions: 1) to walk at a comfortable self-paced speed, normal walk data set;2) to walk at a very slow speed taking shorter steps, slow walk data set; and 3) to walk as comfortably as possible while having the right knee immobilized with a brace in order to simulate limping, limp walk data set. The average number of steps per subject used in the analysis was 9.5 for normal walking, 15.3 for slow walking and 9.5 for limp walking.

The data obtained from the pressure mat was used as ground truth for heel-strike (HS) and toe-off (TO) instances. The proposed method was applied so as to create subject-independent "normal" symbols, as follows. The "normal walk" data sets of 3 randomly chosen individuals were combined, segmented and clustered, resulting in 7 symbols. The centers of these clusters were used to symbolize the remaining data sets into equivalent symbols. All data sets were submitted to the context analysis in the exact same way.

In order to evaluate the performance of the proposed method with regards to previous approaches, a peakdetection based method for detecting HS and TO was implemented [28]. This method was chosen over a more recent work [6] because it is based on the same principles but seems to be less dependent on optimizing parameters, and therefore more robust to different data sets. The method presented in [28] was implemented with a slight modification in order to cope with difference in sensor placement. At each iteration, the interval used to narrow down on the location of the peaks was changed to a window ten-samplewide centered at the previous peak location. All data sets, were submitted to the peak-detection analysis in the exact same way.

The symbol-based symmetry index, $S I_{\text {symb }}$, was computed for all three data sets. In order to evaluate the information conveyed by the symbol-based index, the symmetry index, $S I$, based on [25] was also computed from the ground truth data, i.e. data from the pressure sensitive mat.

\section{Results}

The detected HS and TO instances, both from the proposed method and from the peak detection method, were compared to the ground truth data from the pressure sensitive mat. The resulting mean error and standard deviation for each data set, in seconds, are presented in Tables 1, 2, and 3. The number of false positives and false negatives are shown in Table 4.

\begin{tabular}{|l|l|l|l|}
\hline \multicolumn{4}{|c|}{ Normal walk data } \\
\hline Method & Event & $\begin{array}{l}\text { mean (standard } \\
\text { deviation) }\end{array}$ & $\begin{array}{l}\text { confidence interval } \\
95 \%\end{array}$ \\
\hline \multirow{2}{*}{$\begin{array}{l}\text { Linguistic } \\
\text { approach }\end{array}$} & HS & $0.05(0.05)$ & {$[-0.06,0.15]$} \\
\cline { 2 - 4 } & TO & $0.03(0.02)$ & {$[-0.01,0.07]$} \\
\hline \multirow{2}{*}{$\begin{array}{l}\text { Peak } \\
\text { detection }\end{array}$} & HS & $0.05(0.02)$ & {$[0.02,0.09]$} \\
\cline { 2 - 4 } & TO & $0.04(0.03)$ & {$[-0.01,0.10]$} \\
\hline
\end{tabular}

Table 1. Mean error in seconds and standard deviation for the linguistic approach and peak detection method on the normal walk data set. The average stride time for this data set is $1.01 \mathrm{~s}$.

\begin{tabular}{|l|l|l|l|}
\hline \multicolumn{4}{|c|}{ Slow walk data } \\
\hline \multirow{2}{*}{ Method } & Event & $\begin{array}{l}\text { mean (standard } \\
\text { deviation) }\end{array}$ & $\begin{array}{l}\text { confidence interval } \\
95 \%\end{array}$ \\
\hline $\begin{array}{l}\text { Linguistic } \\
\text { approach }\end{array}$ & HS & $0.10(0.11)$ & {$[-0.12,0.32]$} \\
\cline { 2 - 4 } & TO & $0.09(0.15)$ & {$[-0.20,0.37]$} \\
\hline $\begin{array}{l}\text { Peak } \\
\text { detection }\end{array}$ & HS & $0.30(0.13)$ & {$[0.05,0.55]$} \\
\cline { 2 - 4 } & TO & $0.29(0.18)$ & {$[-0.07,0.66]$} \\
\hline
\end{tabular}

Table 2. Mean error in seconds and standard deviation for the linguistic approach and peak detection method on the slow walk data set. The average stride time for this data set is $1.46 \mathrm{~s}$.

\begin{tabular}{|l|l|l|l|}
\hline \multicolumn{4}{|c|}{ Limp walk data } \\
\hline Method & Event & $\begin{array}{l}\text { mean (standard } \\
\text { deviation) }\end{array}$ & $\begin{array}{l}\text { confidence interval } \\
95 \%\end{array}$ \\
\hline \multirow{2}{*}{$\begin{array}{l}\text { Linguistic } \\
\text { approach }\end{array}$} & HS & $0.10(0.07)$ & {$[-0.03,0.22]$} \\
\cline { 2 - 4 } & TO & $0.09(0.08)$ & {$[-0.07,0.24]$} \\
\hline \multirow{2}{*}{$\begin{array}{l}\text { Peak } \\
\text { detection }\end{array}$} & HS & $0.14(0.05)$ & {$[0.045,0.25]$} \\
\cline { 2 - 4 } & TO & $0.12(0.05)$ & {$[-0.02,0.22]$} \\
\hline
\end{tabular}

Table 3. Mean error in seconds and standard deviation for the linguistic approach and peak detection method on the limp walk data set. The average stride time for this data set is $1.04 \mathrm{~s}$.

Table 1 shows that both the linguistic approach and the peak detection methods may be used to detect HS and TO instances from gait acceleration data during "normal walking" with small errors. Considering the sampling frequency of $50 \mathrm{~Hz}$, the mean errors correspond to approximately 2 accelerometer samples. Table 2 indicates that, 


\begin{tabular}{|l|l|l|l|l|}
\hline \multicolumn{2}{|c|}{ data set } & $\begin{array}{l}\text { normal } \\
\text { walk }\end{array}$ & $\begin{array}{l}\text { slow } \\
\text { walk }\end{array}$ & $\begin{array}{l}\text { limp } \\
\text { walk }\end{array}$ \\
\hline \multirow{2}{*}{$\begin{array}{l}\text { Linguistic } \\
\text { approach }\end{array}$} & false positives & 0 & 0 & 2 \\
\cline { 2 - 5 } & false negatives & 2 & 17 & 6 \\
\hline \multirow{2}{*}{$\begin{array}{l}\text { Peak } \\
\text { detection }\end{array}$} & false positives & 1 & 3 & 2 \\
\cline { 2 - 5 } & false negatives & 18 & 16 & 2 \\
\hline \multicolumn{2}{|c|}{ total number of events } & 144 & 184 & 144 \\
\hline
\end{tabular}

Table 4. Number of false negative and false positive HS and TO detections, for the linguistic approach and peak detection method.

for the slow walk data set, mean errors correspond to approximately $7 \%$ and $20 \%$ of the average stride time for the linguistic approach and for the peak detection method respectively. Table 3 shows that, for the limp walk data set, mean errors correspond to approximately $10 \%$ and $13 \%$ of the average stride time for the linguistic approach and for the peak detection method respectively. Table 4 shows that the peak detection method detected approximately twice as many false positives and negatives than the linguistic approach.

The symmetry indices were calculated for each subject and each data set. The distributions of the symmetry indices within each data set are shown as box-plots in Figure 7. A more detailed plot in Figure 8 shows the corresponding symmetry indices for each subject whithin all three data sets. The symmetry index SI judged the all the data sets as equally symmetric. An ANOVA test indicated that the SI distributions for normal, slow and limp walk were not significantly different, $p=0.3673$. In contrast, the symbol-based symmetry index $S_{\text {symb }}$ consistently judged the normal, slow, and limp data set as progressively less symmetric. An ANOVA test indicated that the $S I_{\text {symb }}$ for normal and limp walk data set distributions were significantly different at a $95 \%$ confidence interval, $p=0.0066$. For all subjects, the $S I_{\text {symb }}$ is consistently higher for the limp data set than for the normal data set, as depicted in Figure 8.

\section{Discussion}

In all cases, the symbolic approach performs equally well or better than the peak detection algorithm. The peak detection results are slightly poorer than the results reporter by [6]. Considering that no parameter optimization was undertaken for this implementation of the peak detection method, results are consistent with previous findings.

Tables 1, 2, and 3 show that the proposed symbolic approach is robust to different data sets. Although the algorithm was designed to analyze normal walking, it provided reasonable estimates of HS and TO for both slow and limp walk data sets. The peak detection method, on the other hand, performed considerably worse on these two data sets. This can be explained by the fact that slow walk generates
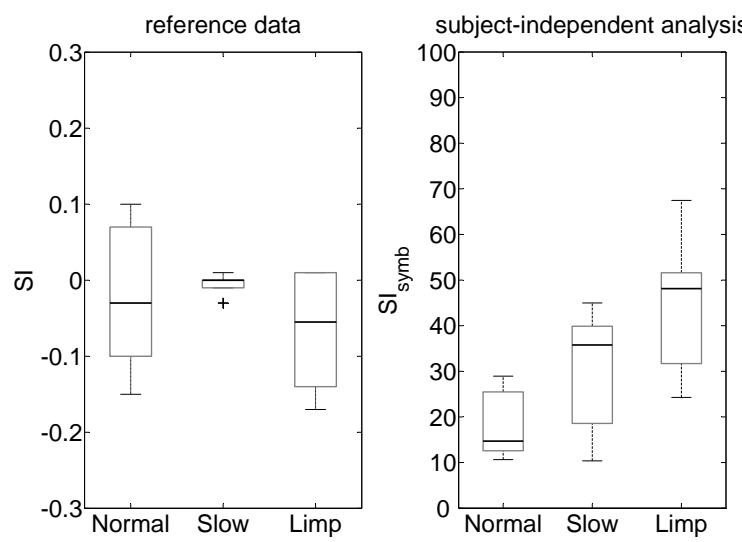

Figure 7. Symmetry indices. The left-hand-side plot shows the traditional symmetry measure which takes into account only average stride times. The right-hand-side plot shows the symbol-based symmetry index, which takes into account static and dynamic characteristics of the signal. The thick horizontal line corresponds to the median; the lower edge of the box corresponds to the lower quartile; the upper edge of the box corresponds to the upper quartile; the lower "whisker" corresponds to the smallest non-outlier value; the upper "whisker" corresponds to the largest non-outlier value; and outlier values are represented with crosses.
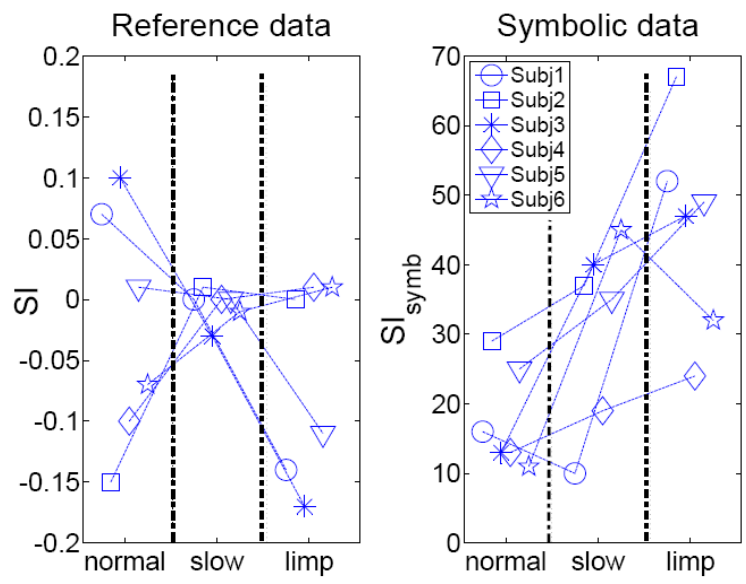

Figure 8. Symmetry indices for each subject. The lefthand-side plot shows the traditional symmetry measure, SI, and the right-hand-side plot shows the symbol-based symmetry index, $S I_{\text {symb }}$, for all three data sets. Each subject is identified with a different marker. For all subjects, the $S I_{\text {symb }}$ is consistently higher for the limp data set than for the normal data set.

much smaller accelerations and milder peaks, which were harder to detect without altering the filtering frequencies and optimizing parameters. Table 4, also indicates that the linguistic approach performs better than the peak detection method. That is because the proposed method searches for the "best match" between symbols and HS or TO, reducing the number of false positives and false negatives. 
Another advantage of the proposed method is its ability to extract more symmetry information than the traditional symmetry index, $S I$. The $S I$ results, illustrated in Figures 7 and 8 show that this index failed to differentiate the different walks according to symmetry. Whereas The symbol based symmetry index $S I_{\text {symb }}$ was able to overlook the temporal symmetry and detect the overall asymmetry of the feet's movements. Slow walk was probably less symmetric than normal walk because the subjects needed to make a conscious effort to change their walking pattern, sometimes oscillating between their normal speed and a slower speed. Although the information conveyed by the symmetry indices are not equivalent, this analysis exemplifies how the $S I$ index fails to extract dynamic information by only taking into account temporal measurements of HS and TO.

The method presented here is totally automatic and does not depend on labeled training data as do most previous linguistic approaches. In addition, the symbolic representation of the signal allows for direct comparison of symbol sequences between subjects, or the evolution of symbol sequences for the same subject over time.

Only one accelerometer per foot was used for the experiments because the envisioned system is to be embedded into the patient's shoes. Although the use of more sensors could improve the accuracy of the analysis, the authors believe that the final system would become too cumbersome to wear. When considering other applications, however, the method can support as many sensors as desired with small modifications to the "context analysis" phase. The intended application should also be taken into account when comparing gait analysis methods. Although the amount of information this method extracts from the signal is minimum compared to motion capture systems, it is as precise and more informative than other currently employed gait analysis systems such as the Gait Rite.

The symbolic approach explored here can be seen as an extension for HAL framework, which enables the use of the methodology presented in [2] on accelerometer data. Other contributions include the use of symbolization in order to extract quantitative gait measurements instead of activity classification; and the creation of a more informative symmetry index.

\section{Conclusion}

This paper presented a linguistic approach to analyze gait using wearable accelerometer sensor nodes. Compared to previous works, the proposed method can, not only detect HS and TO more accurately, but also extract dynamic information from the signal. The characterization of the signal in terms of its dynamics was here exemplified with a novel symbol-based symmetry index. Other contributions of this work include the use of expert knowledge instead of relying on supervised learning techniques and labeled training data, and extracting quantitative measurements from the symbolized data. The symbolization technique suggested here can be used to pre-process acceleration data before using the HAL framework to extract syntax and grammar rules from the symbolized data.

The method proposed here targets wearable sensors systems, which can be used to monitor the quality of gait of older adults and provide insight into their cognitive condition. Orthopedic patients in general, amputees and others suffering from ambulatory dysfunction can also profit from such a system. Future investigations include a clinical study which will validate the proposed method and the symbol-based symmetry index on real patient data.

The proposed method was here applied to accelerometers, however, this approach may be extended to other types of continuous sensor data. Once the characteristics of the system are understood, rules can be designed to detect relevant events in any type of time series, e.g. from ECG to seismographic data.

\section{Acknowledgments}

The author would like to thank: Dr. Misha Pavel and Dr. Holly Jimison, for making the data collection possible; Dr. Fay Horak, for welcoming us into her lab; and Dr. Arash Salarian, for technical support.

\section{References}

[1] O. Beauchet, G. Allali, G. Berrut, C. Hommet, V. Dubost, and F. Assal, "Gait analysis in demented subjects: interests and perspectives," Neuropsychiatric Disease and Treatment, vol. 4, no. 1, pp. 155-160, 2008.

[2] G. Guerra-Filho and Y. Aloimonos, "A language for human action," Computer, vol. 40, no. 5, pp. 42-51, 2007.

[3] M. B. Van Iersel, W. Hoefsloot, M. Munneke, B. R. Bloem, and M. G. M. Olde Rikkert, "Systematic review of quantitative clinical gait analysis in patients with dementia," Zeitschrift fr Gerontologie und Geriatrie, vol. 37, pp. 27-32, 2004.

[4] J. Verghese, C. Wang, R. B. Lipton, R. Holtzer, and $X$. Xue, "Quantitative gait dysfunction and risk of cognitive decline and dementia," Journal of Neurology Neurosurgery and Pshychiatry, vol. 78, no. 9, pp. 929-935, 2007.

[5] D. H. Sutherland, "The evolution of clinical gait analysis, part ii kinematics," Gait and Posture, vol. 16, pp. 159-179, 2002.

[6] R. W. Selles, M. A. G. Formanoy, J. B. J. Bussmann, P. J. Janssens, and H. J. Stam, "Automated estimation of initial and terminal contact timing using accelerometers; development and validation in 
transtibial amputees and controls," IEEE Transactions on Neural Systems and Rehabilitation Engineering, vol. 13, no. 1, pp. $81-88,2005$.

[7] M. J. Mathie, A. C. F. Coster, N. H. Lovell, and B. G. Celler, "Accelerometry: providing an integrated, practical method for long-term, ambulatory monitoring of human movement," Physiological Measurement, vol. 25, no. 2, pp. R1-R20, 2004.

[8] A. Salarian, H. Russmann, F. J. G. Vingerhoets, C. Dehollain, Y. Blanc, P. R. Burkhard, and K. Aminian, "Gait assessment in parkinsons disease: Toward an ambulatory system for long-term monitoring," IEEE Transactions on Biomedical Engineering, vol. 51, no. 8, pp. 1434-1443, 2004.

[9] K. Aminian, B. Najafi, C. Büla, P.-F. Leyvraz, and P. Robert, "Spatio-temporal parameters of gait measured by an ambulatory system using miniature gyroscopes," Journal of Biomechanics, vol. 35, p. 689699, 2002.

[10] J.-A. Lee, S.-H. Cho, J.-W. Lee, K.-H. Lee, and H.-K. Yang, "Wearable accelerometer system for measuring the temporal parameters of gait," 29th Annual International Conference of IEEE-EMBS, Engineering in Medicine and Biology Society, EMBC'07, pp. 483 486, 2007.

[11] J. B. J. Bussmann, L. Damen, and H. J. Stam, "Analysis and decomposition of signals obtained by thighfixed uni-axial accelerometry during normal walking," Medical and Biological Engineering and Computing, vol. 38, pp. 632-638, 2000.

[12] R. Williamson and B. J. Andrews, "Gait event detection for FES using accelerometer and supervised machine learning," IEEE Transactions on Rehabilitation Engineering, vol. 8, no. 3, pp. 312-319, 2000.

[13] M. Sekine, T. Tamura, T. Fujimoto, and Y. Fukui, "Classification of walking pattern using acceleration waveform in elderly people," in Engineering in Medicine and Biology Society, 2000. Proceedings of the 22nd Annual International Conference of the IEEE, vol. 2, 2000.

[14] L. Bao and S. S. Intille, "Activity recognition from user-annotated acceleration data," Pervasive 2004, pp. 1-17, April 2004.

[15] R. K. Ibrahim, E. Ambikairajah, B. G. Celler, and N. H. Lovell, "Time-frequency based features for classification of walking patterns," Digital Signal Processing, 2007 15th International Conference on, pp. 187-190, 2007.

[16] M. N. Nyan, F. E. H. Tay, K. H. W. Seah, and Y. Y. Sitoh, "Classification of gait patterns in the timefrequency domain," Journal of Biomechanics, vol. 39, pp. 2647-2656, 2006.
[17] A. Miller, "Gait event detection using a multilayer neural network," Gait \& Posture, vol. 29, pp. 542$545,2009$.

[18] C. S. Daw, C. E. A. Finney, and E. R. Tracy, "A review of symbolic analysis of experimental data," Review of Scientific Instruments, vol. 74, no. 2, p. 915, 2003.

[19] A. Bobick and J. Davis, "The recognition of human movement using temporal templates," Pattern Analysis and Machine Intelligence, IEEE Transactions on, vol. 23, no. 3, pp. 257-267, 2001.

[20] P. Fihl, M. B. Holte, T. B. Moeslund, and L. Reng, "Action recognition using motion primitives and probabilistic edit distance," in 4th international Conference on Articulated Motion and Deformable $\mathrm{Ob}$ jects, pp. 375-384, 2006.

[21] D. D. Vecchio, R. M. Murray, and P. Perona, "Decomposition of human motion into dynamics based primitives with application to drawing tasks," Automatica, vol. 39, pp. 2085-2098, 2003.

[22] E. Keogh, "Data Mining in Time Series Databases Tutorial," in Proceedings of the IEEE Int. Conference on Data Mining, 2004.

[23] S. Petrovic, "A Comparison Between the Silhouette Index and the Davies-Bouldin Index in Labelling IDS Clusters," in Proceedings of the 11th Nordic Workshop of Secure IT Systems, pp. 53-64, 2006.

[24] A. Sant'Anna, "Improved representation of movements for human motion analysis using mobile inertial sensors: Including expert knowledge to achieve characterization," tech. rep., School of Information Science, Computer and Electrical Engineering, Halmstad University, 2009.

[25] L. Nolan, A. Wit, K. Dudziñski, A. Lees, M. Lake, and M. Wychowañski, "Adjustments in gait symmetry with walking speed in trans-femoral and trans-tibial amputees," Gait \& Posture, vol. 17, no. 2, pp. 142$151,2003$.

[26] shimmer-research.com.

[27] A. McDonough, M. Batavia, F. Chen, S. Kwon, and J. Ziai, "The validity and reliability of the GaitRite system's measurements: A preliminary evaluation," Archives of Physical Medicine and Rehabilitation, vol. 82, no. 3, pp. 419-425, 2001.

[28] K. Aminian, K. Rezakhanlou, E. De Andres, C. Fritsch, P. F. Leyvraz, and P. Robert, "Temporal feature estimation during walking using miniature accelerometers: an analysis of gait improvement after hip arthroplasty," Medical and Biological Engineering and Computing, vol. 37, pp. 686-691, 1999. 